\title{
Extensions of the Minimum Cost Homomorphism Problem
}

\author{
Rustem Takhanov ${ }^{1}$ \\ Department of Computer and Information Science, Linköping University, \\ SE-581 83 Linköping, Sweden \\ takhanov@mail.ru
}

\begin{abstract}
Assume $D$ is a finite set and $R$ is a finite set of functions from $D$ to the natural numbers. An instance of the minimum $R$-cost homomorphism problem $\left(\mathrm{MinHom}_{R}\right)$ is a set of variables $V$ subject to specified constraints together with a positive weight $c_{v r}$ for each combination of $v \in V$ and $r \in R$. The aim is to find a function $f: V \rightarrow D$ such that $f$ satisfies all constraints and $\sum_{v \in V} \sum_{r \in R} c_{v r} r(f(v))$ is minimized. This problem unifies well-known optimization problems such as the minimum cost homomorphism problem and the maximum solution problem, and this makes it a computationally interesting fragment of the valued CSP framework for optimization problems. We parameterize $\mathrm{MinHom}_{R}(\Gamma)$ by constraint languages, i.e. sets $\Gamma$ of relations that are allowed in constraints. A constraint language is called conservative if every unary relation is a member of it; such constraint languages play an important role in understanding the structure of constraint problems. The dichotomy conjecture for $\mathrm{MinHom}_{R}$ is the following statement: if $\Gamma$ is a constraint language, then $\operatorname{MinHom}_{R}(\Gamma)$ is either polynomial-time solvable or NP-complete. For MinHom the dichotomy result has been recently obtained [Takhanov, STACS, 2010] and the goal of this paper is to expand this result to the case of $\mathrm{MinHom}_{R}$ with conservative constraint language. For arbitrary $R$ this problem is still open, but assuming certain restrictions on $R$ we prove a dichotomy. As a consequence of this result we obtain a dichotomy for the conservative maximum solution problem.
\end{abstract}

\section{Introduction}

Constraint satisfaction problems $(C S P)$ and valued constraint satisfaction problems $(V C S P)$ are natural ways of formalizing a large number of computational problems arising in combinatorial optimization, artificial intelligence, and database theory. $C S P$ has the following two equivalent formulations: (1) to find an assignment of values to a given set of variables, subject to constraints on the values that can be assigned simultaneously to specified subsets of variables, and (2) to find a homomorphism between two finite relational structures $A$ and $B . V C S P$ is a "soft" version of $C S P$ where constraint relations are replaced by functions from set of tuples to some totally ordered set with addition operation (for example, rational numbers). A solution is defined as an assignment 
to variables that maximize a functional which is equal to a sum of constraint functions applied to corresponding variables. Applications of $C S P \mathrm{~s}$ and $V C S P \mathrm{~s}$ arise in the propositional logic, database and graph theory, scheduling, biology and many other areas. $C S P$ and its subproblems has been intensively studied by computer scientists and mathematicians since the $70 \mathrm{~s}$, and recently attention has been paid to its modifications such as VCSP. Considerable attention has been given to the case where the constraints are restricted to a given finite set of relations $\Gamma$, called a constraint language $[2,5,12,20]$. For example, when $\Gamma$ is a constraint language over the boolean set $\{0,1\}$ with four ternary predicates $x \vee y \vee z, \bar{x} \vee y \vee z, \bar{x} \vee \bar{y} \vee z, \bar{x} \vee \bar{y} \vee \bar{z}$ we obtain 3-SAT. For every constraint language $\Gamma$, it has been conjectured that $C S P(\Gamma)$ is either in $\mathrm{P}$ or NP-complete [5]. An analogous situation appears in $V C S P$ where the constraint language is defined as a set of "soft" predicates.

We believe that problems like minimum cost homomorphism problem (MinHom) has an intermediate position between $C S P \mathrm{~s}$ and VCSPs which makes their structure important for understanding the relationship between "hard" and "soft" constraints in optimization. In the minimum cost homomorphism problem, we are given variables subject to constraints and, additionally, costs on variable/value pairs. Now, the task is not just to find any satisfying assignment to the variables, but one that minimizes the total cost. In the context of $V C S P$ this is equivalent to addition of "soft" constraints equal to characteristic functions of one element sets. We will consider a weighted version of this problem.

Definition 1. Suppose we are given a finite domain set $A$, a finite constraint language $\Gamma \subseteq \bigcup_{k=1}^{\infty} 2^{A^{k}}$ and a finite set of functions $R \subseteq\{r: A \rightarrow \mathbb{N}\}$. Denote by $\operatorname{MinHom}_{R}(\Gamma)$ the following minimization task:

Instance: A triple $(V, \mathrm{C}, W)$ where

$-V$ is a set of variables;

- $\mathrm{C}$ is a set of constraints, where each constraint $C \in \mathrm{C}$ is a pair $(s, \rho)$, such that

- $s=\left(v_{1}, \ldots, v_{m}\right)$ is a tuple of variables of length $m$, called the constraint scope;

- $\rho$ is an element of $\Gamma$ with arity $m$, called the constraint relation.

- Weights $w_{v r} \in \mathbb{N}, v \in V, r \in R$.

Solution: A function $f$ from $V$ to $A$, such that, for each variable $v \in V$, $f(v) \in A$, and for each constraint $(s, \rho) \in \mathrm{C}$, with $s=\left(v_{1}, \ldots, v_{m}\right)$, the tuple $\left(f\left(v_{1}\right), \ldots, f\left(v_{m}\right)\right)$ belongs to $\rho$.

Measure: $\sum_{v \in V} \sum_{r \in R} w_{v r} r(f(v))$.

Definition 2. For $R^{*}=\left\{e_{i} \mid i \in A\right\}, \operatorname{MinHom}_{R^{*}}(\Gamma)$ is called minimum cost homomorphism problem where $e_{i}: A \rightarrow \mathbb{N}$ denotes a characteristic function of $\{i\} \subseteq A$. 
We will write MinHom instead of MinHom $_{R^{*}}$ for short. MinHom has applications in defence logistics [10] and machine learning [4]. Complete classification of constraint languages $\Gamma$ for which $\operatorname{MinHom}(\Gamma)$ is polynomial-time solvable has recently been obtained in [21]. The question for which directed graphs $H$ the problem MinHom $(\{H\})$ is polynomial-time solvable was considered in [7-11]. Maximum Solution Problem (MaxSol), which is defined analogously to MinHom, but with $A \subseteq \mathbb{N}$ and a functional of the form $\sum_{v \in V} w_{v} f(v)$ to maximize, was investigated in a series of papers [14-16]. It is easy to see that if $n=\max _{s \in A} s+1$ and $R=\{n-x\}$, then $\operatorname{MinHom}_{R}(\Gamma)=\operatorname{MaxSol}(\Gamma)$. In this paper, we will assume that a constraint language $\Gamma$ contains all unary predicates over a domain set $A$ and approach the problem of characterizing the complexity of $\operatorname{MinHom}_{R}(\Gamma)$ in its most general form by algebraic methods. When $R$ satisfies certain conditions, we obtain a dichotomy for $\operatorname{MinHom}_{R}(\Gamma)$, i.e., if $\operatorname{MinHom}_{R}(\Gamma)$ is not polynomial-time solvable, then it is NP-hard. As a consequence, we obtain a dichotomy for conservative MaxSol.

In Section 2, we present some preliminaries together with results connecting the complexity of $\mathrm{MinHom}_{R}$ with conservative algebras. The main dichotomy theorem is stated in Section 3 and its proof is divided into several parts which can be found in Sections 4-6. Finally, in Section 7 we present directions for future research.

\section{Algebraic structure of tractable constraint languages}

Recall that an optimization problem $A$ is called NP-hard if some NP-complete language can be recognized in polynomial time with the aid of an oracle for $A$. We assume that $P \neq N P$.

Definition 3. Suppose we are given a finite set $A$ and a constraint language $\Gamma \subseteq \bigcup_{k=1}^{\infty} 2^{A^{k}}$. The language $\Gamma$ is said to be $R$-tractable if, for every finite subset $\Gamma^{\prime} \subseteq \Gamma$, the task $\mathrm{MinHom}_{R}\left(\Gamma^{\prime}\right)$ is polynomial-time solvable, and $\Gamma$ is called $R$-NP-hard if there is a finite subset $\Gamma^{\prime} \subseteq \Gamma$, such that the task MinHom $_{R}\left(\Gamma^{\prime}\right)$ is NP-hard.

First, we will state some standard definitions from universal algebra.

Definition 4. Let $\rho \subseteq A^{m}$ and $f: A^{n} \rightarrow A$. We say that the function (operation) $f$ preserves the predicate $\rho$ if, for every $\left(x_{1}^{i}, \ldots, x_{m}^{i}\right) \in \rho, 1 \leq i \leq n$, we have that $\left(f\left(x_{1}^{1}, \ldots, x_{1}^{n}\right), \ldots, f\left(x_{m}^{1}, \ldots, x_{m}^{n}\right)\right) \in \rho$.

For a constraint language $\Gamma$, let $\operatorname{Pol}(\Gamma)$ denote the set of operations preserving all predicates in $\Gamma$. Throughout the paper, we let $A$ denote a finite domain and $\Gamma$ a constraint language over $A$. We assume the domain $A$ to be finite.

Definition 5. A constraint language $\Gamma$ is called a relational clone if it contains every predicate expressible by a first-order formula involving only 
a) predicates from $\Gamma \cup\left\{={ }^{A}\right\}$;

b) conjunction; and

c) existential quantification.

First-order formulas involving only conjunction and existential quantification are often called primitive positive ( $p p$ ) formulas. For a given constraint language $\Gamma$, the set of all predicates that can be described by pp-formulas over $\Gamma$ is called the closure of $\Gamma$ and is denoted by $\langle\Gamma\rangle$.

For a set of operations $F$ on $A$, let $\operatorname{Inv}(F)$ denote the set of predicates preserved under the operations of $F$. Obviously, $\operatorname{Inv}(F)$ is a relational clone. The next result is well-known $[1,6]$.

Theorem 1. For a constraint language $\Gamma$ over a finite set $A,\langle\Gamma\rangle=$ Inv $(\operatorname{Pol}(\Gamma))$.

Theorem 1 tells us that the Galois closure of a constraint language $\Gamma$ is equal to the set of all predicates that can be obtained via pp-formulas from the predicates in $\Gamma$. We will omit the proof of the following standard theorem.

Theorem 2. For any finite constraint language $\Gamma$, finite $\Gamma^{\prime} \subseteq\langle\Gamma\rangle$ and finite $R \subseteq\{r: A \rightarrow \mathbb{N}\}$ there is a polynomial time reduction from $\operatorname{MinHom}_{R}\left(\Gamma^{\prime}\right)$ to $\operatorname{MinHom}_{R}(\Gamma)$.

The previous theorem tells us that the complexity of $\operatorname{MinHom}_{R}(\Gamma)$ is basically determined by $\operatorname{Inv}(\operatorname{Pol}(\Gamma))$, i.e., by $\operatorname{Pol}(\Gamma)$. That is why we will be concerned with the classification of sets of operations $F$ for which $\operatorname{Inv}(F)$ is a tractable constraint language.

Definition 6. An algebra is an ordered pair $\mathcal{A}=(A, F)$ such that $A$ is a nonempty set (called a universe) and $F$ is a family of finitary operations on A. An algebra with a finite universe is referred to as a finite algebra.

Definition 7. An algebra $\mathcal{A}=(A, F)$ is called $R$-tractable if $\operatorname{Inv}(F)$ is a $R$ tractable constraint language and $\mathcal{A}$ is called $R$-NP-hard if $\operatorname{Inv}(F)$ is an $R$-NPhard constraint language.

For $B \subseteq A$, define $R_{B}=\left\{\left.f\right|_{B} \mid f \in R\right\}$, where $\left.f\right|_{B}$ is a restriction of $f$ on a set $B$. We will use the term MinHom-tractable (NP-hard) instead of $\left\{e_{i} \mid i \in A\right\}$ tractable (NP-hard) and, in case $A=\{0,1\}$, the term min-tractable (NP-hard) instead of $\{x\}$-tractable (NP-hard).

We only need to consider a very special type of algebras, so called conservative algebras.

Definition 8. An algebra $\mathcal{A}=(A, F)$ is called conservative if for every operation $f \in F$ we have that $f\left(x_{1}, \ldots, x_{n}\right) \in\left\{x_{1}, \ldots, x_{n}\right\}$.

Since we assume that $\Gamma$ is a constraint language with all unary relations over the domain set $A$, then $\mathcal{A}=(A, \operatorname{Pol}(\Gamma))$ is conservative. Besides conservativeness of constraint languages we will make some additional restrictions on function sets $R$. 
Definition 9. Suppose we are given a finite set of functions $R \subseteq\{r: A \rightarrow \mathbb{N}\}$. Denote by $G(R)=(A, E(R))$ a directed graph with a set of vertices $A$ and an edge set $E(R)=\{(a, b) \mid \exists r \in R r(a)>r(b)\}$. The $U G(R)$ is the graph $G(R)$ with all edges considered as undirected. We will call $G$ a preference graph and $U G$ an undirected preference graph.

In the sequel, we will assume that a graph $U G$ is complete. It is easy to see that $U G\left(\left\{e_{i} \mid i \in A\right\}\right), U G(\{n-x\})$ are complete and our results can be applied to MinHom and MaxSol.

\section{Boolean case and the necessary local conditions}

The first step to understand the structure of $R$-tractable algebras is to understand the boolean case. Well-known structure of boolean clones [18] helps us to prove the following theorem.

Theorem 3. A boolean clone $H$ is MinHom-tractable if either $\{x \wedge y, x \vee y\} \subseteq$ $H$ or $\{(x \wedge \bar{y}) \vee(\bar{y} \wedge z) \vee(x \wedge z)\} \subseteq H$, where $\bar{x}, x \wedge y, x \vee y$ denote negation, conjunction and disjunction. Otherwise, $H$ is MinHom-NP-hard. A conservative boolean clone $H$ is min-tractable if either $\{x \wedge y\} \subseteq H$ or $\{(x \wedge \bar{y}) \vee(\bar{y} \wedge z) \vee(x \wedge z)\} \subseteq H$. Otherwise, $H$ is min-NP-hard.

In the proof of Theorem 3 we will need the following definition.

Definition 10. A constraint language $\Gamma$ over $\{0,1\}$ is called a MinHom(min)maximal constraint language if it is conservative MinHom(min)-tractable and is not contained in any other conservative MinHom(min)-tractable languages.

We identify all MinHom(min)-maximal constraint languages using Post's classification [18]. Via Theorems 1,2 we conclude that every MinHom(min)maximal constraint language corresponds to some conservative functional clone. In the case $A=\{0,1\}$, there is a countable number of conservative clones: we list them below according to the table on page 76 [17].

Lemma 1. The relational clones Inv $\left(M_{01}\right)$ and Inv $\left(S_{01}\right)$ are MinHommaximal constraint languages. Every other constraint language given in the table that is not contained in any of these two is MinHom-NP-hard. The relational clones Inv $\left(K_{01}\right)$ and Inv $\left(S_{01}\right)$ are min-maximal constraint languages. Every other constraint language given in the table that is not contained in any of these two is min-NP-hard. 
Proof. For every row, the closure of the predicates given is equal to the set of all predicates preserved under the functions of the corresponding clone.

$$
\begin{array}{cc}
T_{01} & x=0, x=1 \\
M_{01} & x=0, x=1, x_{1} \leq x_{2} \\
S_{01} & x=0, x_{1} \neq x_{2} \\
S M & x_{1} \neq x_{2}, x_{1} \leq x_{2} \\
L_{01} & x=1, x_{1} \oplus x_{2} \oplus x_{3}=0 \\
U_{01} & x=0, x=1, x_{1}=x_{2} \vee x_{1}=x_{3} \\
K_{01} & x=0, x=1, x_{1}=x_{2} x_{3} \\
D_{01} & x=0, x=1, x_{1}=x_{2} \vee x_{3} \\
I_{1}^{m} & x=1, x_{1} x_{2} \ldots x_{m}=0 \\
M I_{1}^{m} & x=1, x_{1} \leq x_{2}, x_{1} x_{2} \ldots x_{m}=0 \\
O_{0}^{m} & x=0, x_{1} \vee x_{2} \vee \ldots \vee x_{m}=1 \\
M O_{0}^{m} & x=0, x_{1} \leq x_{2}, x_{1} \vee x_{2} \vee \ldots \vee x_{m}=1
\end{array}
$$

where $x \oplus y=x+y(\bmod 2)$.

The class $\operatorname{Inv}\left(T_{01}\right)$ is MinHom(min)-tractable, since it contains only two simple unary predicates $\{0\}$ and $\{1\}$. As we will see later, it cannot be MinHom(min)-maximal since it is included in other MinHom(min)-tractable constraint languages.

Let us prove that Inv $\left(M_{01}\right)$ and Inv $\left(S_{01}\right)$ are MinHom-tractable. By Theorem 2, it is equivalent to polynomial solvability of $\operatorname{MinHom}\left(\left\{\{0\},\{1\},\left\{\left(x_{1}, x_{2}\right) \mid x_{1} \leq x_{2}\right\}\right\}\right)$ and MinHom $\left(\left\{\{0\},\left\{\left(x_{1}, x_{2}\right) \mid x_{1} \neq x_{2}\right\}\right\}\right)$, because the classes Inv $\left(M_{01}\right)$ and $\operatorname{Inv}\left(S_{01}\right)$ are the closures of those sets. We will skip the proof since it can be found in [13] (in this paper boolean MinHom called Max AW Ones). It is easy to see that MinHom-tractability implies min-tractability of those classes.

Let us show that all the classes in the table, except Inv $\left(M_{01}\right)$, Inv $\left(S_{01}\right)$ and $\operatorname{Inv}\left(T_{01}\right)$, are MinHom-NP-hard, and all the classes, except $\operatorname{Inv}\left(M_{01}\right)$, $\operatorname{Inv}\left(S_{01}\right), \operatorname{Inv}\left(T_{01}\right), \operatorname{Inv}\left(K_{01}\right)$ and $\operatorname{Inv}\left(M I_{1}^{2}\right), \ldots, \operatorname{Inv}\left(M I_{1}^{\infty}\right)$, where $M I_{1}^{\infty}=$ $\bigcup_{m=1}^{\infty} M I_{1}^{m}$, are min-NP-hard. Since,

$$
\begin{aligned}
& x_{1} \vee x_{2}=\exists x_{3}\left[x_{1} \neq x_{3}\right] \wedge\left[x_{3} \leq x_{2}\right] \\
& x_{1} \vee x_{2}=\exists x_{3}\left[x_{3}=1\right] \wedge\left[x_{3}=x_{1} \vee x_{3}=x_{2}\right] \\
& \overline{x_{1}} \vee \overline{x_{2}}=\exists x_{3}\left[x_{3}=0\right] \wedge\left[x_{3}=x_{1} x_{2}\right] \\
& x_{1} \vee x_{2}=\exists x_{3}\left[x_{3}=1\right] \wedge\left[x_{3}=x_{1} \vee x_{2}\right] \\
& \overline{x_{1}} \vee \overline{x_{2}}=\exists x_{3} \ldots x_{m}\left[x_{1} x_{2} \ldots x_{m}=0\right] \wedge\left[x_{2}=x_{3}\right] \wedge \ldots \wedge\left[x_{m-1}=x_{m}\right] \\
& x_{1} \vee x_{2}=\exists x_{3} \ldots x_{m}\left[x_{1} \vee x_{2} \vee \ldots \vee x_{m}=1\right] \wedge\left[x_{2}=x_{3}\right] \wedge \ldots \wedge\left[x_{m-1}=x_{m}\right]
\end{aligned}
$$

we

$\left\{\left(x_{1}, x_{2}\right) \mid x_{1} \vee x_{2}\right\} \in \operatorname{Inv}(S M), \operatorname{Inv}\left(U_{01}\right), \operatorname{Inv}\left(D_{01}\right), \operatorname{Inv}\left(O_{0}^{m}\right), \operatorname{Inv}\left(M O_{0}^{m}\right)$ and $\left\{\left(x_{1}, x_{2}\right) \mid \overline{x_{1}} \vee \overline{x_{2}}\right\} \in \operatorname{Inv}\left(K_{01}\right), \operatorname{Inv}\left(I_{1}^{m}\right), \operatorname{Inv}\left(M I_{1}^{m}\right)$.

We first prove that $\operatorname{MinHom}_{\{x\}}\left(\left\{\left\{\left(x_{1}, x_{2}\right) \mid x_{1} \vee x_{2}\right\}\right\}\right)$ is NP-hard. Suppose an instance of this problem consists of an undirected graph $G=(V, E)$ where each vertex is considered as a variable. For each pair of variables 
$(u, v) \in E$, we require their assignments to satisfy $u=1$ or $v=1$. It is easy to see that for any such assignment $f$, the set $\{x \mid f(x)=0\}$ is independent in the graph $G$. Furthermore, for any independent set $S$ in the graph $G$, $g(x)=[x \notin S]$ is a satisfying assignment. If we define $w_{i}=1$ for $i \in V$, then MinHom $_{\{x\}}$ is equivalent to finding a maximum independent set. This implies that $\operatorname{MinHom}_{\{x\}}\left(\left\{\left\{\left(x_{1}, x_{2}\right) \mid x_{1} \vee x_{2}\right\}\right\}\right)$ is NP-hard, since finding independent sets of maximal size is an NP-hard problem.

Therefore, Inv $(S M)$, Inv $\left(U_{01}\right), \operatorname{Inv}\left(D_{01}\right), \operatorname{Inv}\left(O_{0}^{m}\right), \operatorname{Inv}\left(M O_{0}^{m}\right)$ are minNP-hard, and, consequently, MinHom-NP-hard.

Classes $\operatorname{Inv}\left(K_{01}\right)$, Inv $\left(I_{1}^{m}\right)$, Inv $\left(M I_{1}^{m}\right)$ are MinHom-NP-hard also since MinHom $\left(\left\{\left\{\left(x_{1}, x_{2}\right) \mid x_{1} \vee x_{2}\right\}\right\}\right)$ and MinHom $\left(\left\{\left\{\left(x_{1}, x_{2}\right) \mid \overline{x_{1}} \vee \overline{x_{2}}\right\}\right\}\right)$ are equivalent. Let us show that these classes are min-tractable. It is easy to see that $\operatorname{Inv}\left(K_{01}\right)$ contains $\operatorname{Inv}\left(I_{1}^{m}\right)$, Inv $\left(M I_{1}^{m}\right)$ and $\wedge \in K_{01}$. Indeed, any constraint satisfaction problem with predicates from $\operatorname{Inv}\left(K_{01}\right)$ can be solved by local 1-consistency algorithm and a solution is an assignment of every variable to a minimum of its allowed values. Obviously, the same algorithm solves MinHom $_{\{x\}}\left(\operatorname{Inv}\left(K_{01}\right)\right)$.

It remains to prove min-NP-hardness of $\operatorname{Inv}\left(L_{01}\right)$ which will also show its MinHom-NP-hardness. First we will show that using an algorithm for $\operatorname{MinHom}_{\{x\}}\left(\left\{\left(x_{1}, x_{2}, x_{3}\right) \mid x_{1} \oplus x_{2} \oplus x_{3}=1\right\}\right)$ as an oracle, we can solve MaxCUT in polynomial time. Since we have that $x_{1} \oplus x_{2} \oplus x_{3}=1 \Leftrightarrow \exists y, z x_{1} \oplus x_{2} \oplus y=$ $0 \& y \oplus x_{3} \oplus z=0 \& z=1$, we will conclude that $\operatorname{Inv}\left(L_{01}\right)$ is min-NP-hard.

Let $G=(V, E)$ be a graph and introduce variables $x_{i j}, y_{i}, y_{j}, i, j \in V$. A system of equations $x_{i j} \oplus y_{i} \oplus y_{j}=1, i, j \in V$ can be viewed as an instance of $\operatorname{MinHom}_{\{x\}}\left(\left\{\left(x_{1}, x_{2}, x_{3}\right) \mid x_{1} \oplus x_{2} \oplus x_{3}=1\right\}\right)$. It is easy to see that arbitrary boolean vector $\bar{y}=\left(y_{1}, \ldots, y_{|V|}\right)$ defines a single solution $x_{i j}=y_{i} \oplus y_{j} \oplus 1, i, j \in V$ of the system. Vector $\bar{y}$ can be considered as the cut $\left\{i \mid y_{i}=1\right\} \subseteq V$ and the value $\sum_{i j}\left(1-x_{i j}\right)$ is equal to the doubled cost of the cut. Then Max-CUT is polynomially reduced to minimizing $\sum_{i j} x_{i j}$ which is equivalent to $\operatorname{MinHom}_{\{x\}}$.

Only two classes Inv $\left(M_{01}\right)$ and Inv $\left(S_{01}\right)$ are left as candidates for MinHommaximality. Since they are not included in each other, they are both maximal. The same argument shows that $\operatorname{Inv}\left(K_{01}\right)$ and $\operatorname{Inv}\left(S_{01}\right)$ are min-maximal.

Proof of Theorem 12. Obviously, $\{\wedge, \vee\}$ is a basis of $M_{01},\{\wedge\}$ is a basis of $K_{01}$ and $\{(x \wedge \bar{y}) \vee(\bar{y} \wedge z) \vee(x \wedge z)\}$ is a basis of $S_{01}$. By Lemma 1 we conclude the statement of the theorem.

Definition 11. We call a pair of vertices $\{a, b\}$ in $G(R)$ a MinHom-pair if they have arcs in both directions and we call $\{a, b\}$ a min-pair if they have an arc in one direction only.

Suppose $f \in F$. By $\underset{b}{\stackrel{a}{\downarrow}} f$, we mean $a \neq b$ and $f(a, b)=f(b, a)=b$. Guided by Theorem 3 we obtain the following definition. 
Definition 12. Let $F$ be a conservative functional clone over $A$ and $R \subseteq$ $\{r: A \rightarrow \mathbb{N}\}$. We say that $F$ satisfies the necessary local conditions for $R$ if and only if

(1) for every MinHom-pair $\{a, b\}$ of $G(R)$, either

(1.a) there exists $f_{1}, f_{2} \in F$ s.t. $\underset{b}{\downarrow} f_{1}$ and $\underset{b}{\uparrow} f_{2}$; or

(1.b) there exists $f \in F$ s.t. $\left.f\right|_{\{a, b\}}(x, x, y)=\left.f\right|_{\{a, b\}}(y, x, x)=$ $\left.f\right|_{\{a, b\}}(y, x, y)=y$,

(2) for every min-pair $\{a, b\}$ of $G(R)$ such that $(a, b) \in E(R)$, either

(2.a) there exists $f \in F$ s.t. $\stackrel{\downarrow}{\downarrow} f$; or

(2.b) there exists $f \in \stackrel{b}{F}$ s.t. $\left.f\right|_{\{a, b\}}(x, x, y)=\left.f\right|_{\{a, b\}}(y, x, x)=$ $\left.f\right|_{\{a, b\}}(y, x, y)=y$.

Theorem 4. Suppose $F$ is a conservative functional clone. If $F$ is $R$-tractable and $U G(R)$ is complete, then it satisfies the necessary local conditions for $R$. If $F$ does not satisfy the necessary local conditions for $R$, then it is $R$-NP-hard.

As in case of MinHom, the necessary local conditions are not sufficient for $R$-tractability of a conservative clone. Let $M=$ $\left\{B|B \subseteq A|, B|=2, F|_{B}\right.$ contains 2 different binary commutative functions $\}$, $M^{o}=\{(a, b) \mid\{a, b\} \in M\}$ and $\bar{M}=\{B|B \subseteq A| B \mid,=2\} \backslash M$.

Introduce an undirected graph without loops $T_{F}^{R}=\left(M^{o} \cap E(R), P\right)$ where $P=\left\{\langle(a, b),(c, d)\rangle \mid(a, b),(c, d) \in M^{o} \cap E(R)\right.$, there is no $\left.\left.f \in F \underset{\substack{a c \\ b}}{b d}\right\}\right\}$.

Theorem 5. Suppose $F$ satisfy the necessary local conditions for $R$ and $U G(R)$ is complete. If the graph $T_{F}^{R}=\left(M^{o} \cap E(R), P\right)$ is bipartite, then $F$ is $R$ tractable. Otherwise, $F$ is $R$-NP-hard.

A proof for NP-hard case of Theorem 5 will be omitted since it is basically the same as in case of MinHom [21].

\section{Multi-sorted MinHom and its tractable case}

Definition 13. For any collection of sets $\mathrm{A}=\left\{A_{i} \mid i \in I\right\}$, and any list of indices $i_{1}, \ldots, i_{m} \in I$, a subset $\rho$ of $A_{i_{1}} \times \ldots \times A_{i_{m}}$, together with the list $\left(i_{1}, \ldots, i_{m}\right)$, will be called a multi-sorted relation over $\mathrm{A}$ with arity $m$ and signature $\left(i_{1}, \ldots, i_{m}\right)$. For any such relation $\rho$, the signature of $\rho$ will be denoted $\sigma(\rho)$.

Definition 14. Let $\Gamma$ be a set of multi-sorted relations over a collection of sets $\mathrm{A}=\left\{A_{i} \mid i \in I\right\}$. The multi-sorted MinHom problem over $\Gamma$, denoted MMinHom $(\Gamma)$, is defined to be the minimization problem with

Instance: $A$ quadruple $(V, \delta, \mathrm{C}, W)$ where

$-V$ is a set of variables;

$-\delta$ is a mapping from $V$ to $I$, called the domain function; 
- $\mathrm{C}$ is a set of constraints, where each constraint $C \in \mathrm{C}$ is a pair $(s, \rho)$, such that

- $s=\left(v_{1}, \ldots, v_{m}\right)$ is a tuple of variables of length $m$, called the constraint scope;

- $\rho$ is an element of $\Gamma$ with arity $m$ and signature $\left(\delta\left(v_{1}\right), \ldots, \delta\left(v_{m}\right)\right)$, called the constraint relation.

- Weights $w_{v a} \in \mathbb{N}, v \in V, a \in A_{\delta(v)}$.

Solution: A function $f$ from $V$ to $\bigcup_{i \in I} A_{i}$, such that, for each variable $v \in V$, $f(v) \in A_{\delta(v)}$, and for each constraint $(s, \rho) \in \mathrm{C}$, with $s=\left(v_{1}, \ldots, v_{m}\right)$, the tuple $\left(f\left(v_{1}\right), \ldots, f\left(v_{m}\right)\right)$ belongs to $\rho$.

Measure: $\sum_{v \in V} w_{v f(v)}$.

We can consider any multi-sorted relation $\rho$ over $\mathrm{A}=\left\{A_{i} \mid i \in I\right\}$ as an ordinary relation $\rho^{\mathrm{A}}$ over a set $\bigcup_{i \in I} A_{i}$ where $A_{i}, i \in I$ are considered to be disjoint. If $\Gamma$ is a set of multi-sorted relations over $\mathrm{A}=\left\{A_{i} \mid i \in I\right\}$, then $\Gamma^{\mathrm{A}}$ denotes a set of relations of $\Gamma$ considered as relations over $\bigcup_{i \in I} A_{i}$. It is easy to see that $\operatorname{MMinHom}(\Gamma)$ is equivalent to $\operatorname{MinHom}\left(\Gamma^{\mathrm{A}}\right)$.

Definition 15. A set of multi-sorted relations over A, $\Gamma$, is said to be MinHomtractable, if $\Gamma^{\mathrm{A}}$ is MinHom-tractable. A set of multi-sorted relations over $\mathrm{A}, \Gamma$, is said to be MinHom-NP-complete, if $\Gamma^{\mathrm{A}}$ is MinHom-NP-complete.

Definition 16. Let $\mathrm{A}$ be a collection of sets. An $n$-ary multi-sorted operation $t$ on $\mathrm{A}$ is defined by a collection of interpretations $\left\{t^{A} \mid A \in \mathrm{A}\right\}$, where each $t^{A}$ is an n-ary operation on the corresponding set $A$. The multi-sorted operation $t$ on $A$ is said to be a polymorphism of a multi-sorted relation $\rho$ over $\mathrm{A}$ with signature $(\delta(1), \ldots, \delta(m))$ if, for any $\left(a_{11}, \ldots, a_{m 1}\right), \ldots,\left(a_{1 n}, \ldots, a_{m n}\right) \in \rho$, we have

$$
t\left(\begin{array}{ccc}
a_{11} & \cdots & a_{1 n} \\
\vdots & & \vdots \\
a_{m 1} & \cdots & a_{m n}
\end{array}\right)=\left(\begin{array}{c}
t^{\delta(1)}\left(a_{11}, \ldots, a_{1 n}\right) \\
\vdots \\
t^{\delta(m)}\left(a_{m 1}, \ldots, a_{m n}\right)
\end{array}\right) \in \rho
$$

For any given set of multi-sorted relations $\Gamma, M P o l(\Gamma)$ denotes the set of multi-sorted operations which are polymorphisms of every relation in $\Gamma$.

Definition 17. Suppose a set of operations $H$ over $D$ is conservative and $B \subseteq\{\{x, y\} \mid x, y \in D, x \neq y\}$. A pair of binary operations $\phi, \psi \in H$ is called $a$ tournament pair on $B$, if $\forall\{x, y\} \in B \phi(x, y)=\phi(y, x), \psi(x, y)=$ $\psi(y, x), \phi(x, y) \neq \psi(x, y)$ and for arbitrary $\{x, y\} \in \bar{B}, \phi(x, y)=x, \psi(x, y)=$ $x$. An operation $m \in H$ is called arithmetical on $B$, if $\forall\{x, y\} \in B m(x, x, y)=$ $m(y, x, x)=m(y, x, y)=y$.

The following theorem is a simple consequence of the main result of [21]. 
Theorem 6. Let $\Gamma$ be a constraint language over $A$ containing all unary relations and $B \subseteq\{\{a, b\} \mid a, b \in A, a \neq b\}$. If Pol $(\Gamma)$ contains operations $\phi, \psi, m$ such that

- $\phi, \psi$ is a tournament pair on $B$,

$-m$ is arithmetical on $\bar{B}$,

then $\Gamma$ is MinHom-tractable.

The following theorem is a generalization of the previous one.

Theorem 7. Let $\Gamma$ be a set of multi-sorted relations over a collection of finite sets $\mathrm{A}=\left\{A_{1}, \ldots, A_{n}\right\}$ containing all unary multi-sorted relations. Assume that $B_{i} \subseteq\left\{\{a, b\} \mid a, b \in A_{i}, a \neq b\right\}$. If $\mathrm{MPol}(\Gamma)$ contains a multi-sorted operations $\phi, \psi, m$ such that

- $\phi^{A_{i}}, \psi^{A_{i}}$ is a tournament pair on $B_{i}$,

- $m^{A_{i}}$ is arithmetical on $\overline{B_{i}}$,

then $\Gamma^{\mathrm{A}}$ is MinHom-tractable.

Proof. Denote $G=\bigcup_{i=1}^{n} A_{i}$. It is easy to see that we can define operations $\phi^{\prime}, \psi^{\prime}$ : $G^{2} \rightarrow G$ and $m^{\prime}: G^{3} \rightarrow G$ such that $\left.\phi^{\prime}\right|_{A_{i}}=\phi^{A_{i}},\left.\psi^{\prime}\right|_{A_{i}}=\psi^{A_{i}},\left.m^{\prime}\right|_{A_{i}}=m^{A_{i}}$ and $\phi^{\prime}, \psi^{\prime}$ is a tournament pair on $\bigcup_{i=1}^{n} B_{i} \cup\left\{\{a, b\} \mid a \in A_{i}, b \in A_{j}, i \neq j\right\}$. Then $\phi^{\prime}, \psi^{\prime}, m^{\prime} \in \operatorname{Pol}\left(\Gamma^{\mathrm{A}}\right)$ which by Theorem 6 means that $\Gamma^{\mathrm{A}}$ is MinHom-tractable.

\section{$5 \quad$ Structure of $\boldsymbol{R}$-tractable algebras}

Definition 18. Suppose a set of operations $H$ over $D$ is conservative and $O \subseteq$ $\{(x, y) \mid x, y \in D, x \neq y\}$. A pair of binary operations $\phi, \psi \in H$ is called a weak tournament pair on $O$, if

(1) $\forall a, b$ such that $(a, b),(b, a) \in O: \underset{b}{\underset{b}{\downarrow} \phi, \uparrow} \underset{b}{a} \underset{b}{a} \underset{b}{\stackrel{a}{\uparrow} \phi, \underset{b}{\downarrow} \psi}$;

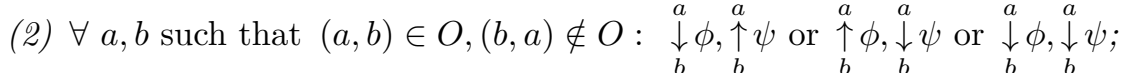

(3) $\forall a, b$ such that $(a, b) \notin O,(b, a) \notin O:\left.\phi\right|_{\{a, b\}}(x, y)=x,\left.\psi\right|_{\{a, b\}}(x, y)=y$.

For a binary operation $f \quad F \quad$ define $\operatorname{Com}(f)=\left\{\{a, b\}:\left.f\right|_{\{a, b\}}\right.$ is commutative $\}$. Consider any binary operation $f_{\max } \in F$ which has maximal set $\operatorname{Com}\left(f_{\max }\right)$, i.e. there is no $f \in F$ such that $\operatorname{Com}\left(f_{\max }\right) \subset \operatorname{Com}(f)$. Since for any binary operations $a, b, \operatorname{Com}(a(x, y)) \cup$ $\operatorname{Com}(b(x, y))=\operatorname{Com}(a(b(x, y), b(y, x)))$, we conclude that $\operatorname{Com}\left(f_{\max }\right)=$ $\bigcup_{f \in F} \operatorname{Com}(f)$. Define $\operatorname{Com}^{o}\left(f_{\max }\right)=\left\{(a, b) \mid\{a, b\} \in \operatorname{Com}\left(f_{\max }\right)\right\}$.

Theorem 8. If $F$ satisfies the necessary local conditions for $R, U G(R)$ is complete and $T_{F}^{R}=\left(M^{o} \cap E(R), P\right)$ is bipartite, then there is a pair $\phi, \psi \in F$ which is a weak tournament pair on $\operatorname{Com}^{o}\left(f_{\max }\right) \cap E(R)$. 
Proof. Let $M_{1}, M_{2}$ denote a partitioning of vertices of the bipartite graph $T_{F}^{R}$. Then, for every $(a, b),(c, d) \in M_{1}$, there is a function $\phi \in F: \stackrel{a c}{\downarrow \downarrow} \phi$. Let us prove by induction that for every $\left(a_{1}, b_{1}\right),\left(a_{2}, b_{2}\right), \ldots,\left(a_{n}, b_{n}\right) \in M_{1}$, there is a

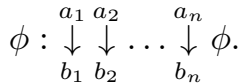

The base of induction $n=2$ is obvious. Let $\left(a_{1}, b_{1}\right),\left(a_{2}, b_{2}\right), \ldots$, $\left(a_{n+1}, b_{n+1}\right) \in M_{1}$ be given. By the induction hypothesis, there are $\phi_{1}, \phi_{2}, \phi_{3} \in$

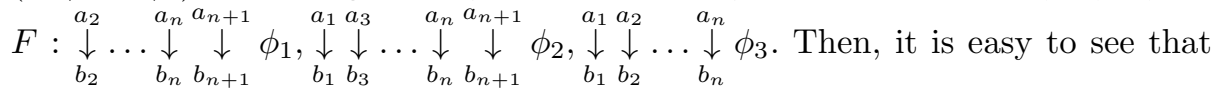
$a_{1} \quad a_{n} a_{n+1}$

$\underset{b_{1}}{\downarrow} \ldots \underset{b_{n}}{\downarrow} b_{n+1} \downarrow \phi_{3}\left(\phi_{1}(x, y), \phi_{2}(x, y)\right)$ which completes the induction proof.

The analogous statement can be proved for $M_{2}$. So it follows from the proof

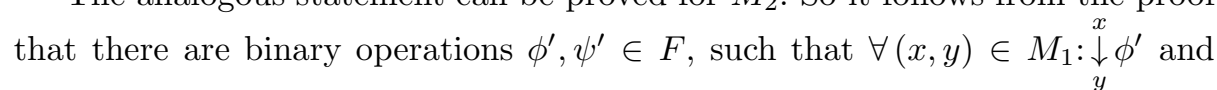
$\forall(x, y) \in M_{2}: \underset{y}{\downarrow} \psi^{\prime}$.

If $(a, b),(b, a) \in \operatorname{Com}^{o}\left(f_{\max }\right) \cap E(R)$, then the necessary local conditions for $C$ give that $\{a, b\} \in M$. Moreover, $(a, b),(b, a) \in M^{o} \cap E(R)$ are always in different partitions of $T_{F}^{R}$. Consequently, $\phi^{\prime}, \psi^{\prime}$ satisfy the first property of a weak tournament pair on $\operatorname{Com}^{o}\left(f_{\max }\right) \cap E(R)$. Our goal is to construct a pair of operations that satisfy other two properties.

Consider operations $\phi^{\prime \prime}(x, y)=f_{\max }\left(\phi^{\prime}(x, y), \phi^{\prime}(y, x)\right)$ and $\psi^{\prime \prime}(x, y)=$ $f_{\max }\left(\psi^{\prime}(x, y), \psi^{\prime}(y, x)\right)$. Since for any $B \in \operatorname{Com}\left(\phi^{\prime}\right),\left.\phi^{\prime \prime}\right|_{B}=\left.\phi^{\prime}\right|_{B}$ and $\left.\psi^{\prime \prime}\right|_{B}=$ $\left.\psi^{\prime}\right|_{B}$, we obtain that $\phi^{\prime \prime}, \psi^{\prime \prime}$ satisfy the first property of a weak tournament pair on $\operatorname{Com}^{\circ}\left(f_{\max }\right) \cap E(R)$, too. Moreover, $\phi^{\prime \prime}, \psi^{\prime \prime}$ are commutative on $\operatorname{Com}\left(f_{\max }\right)$. For any two elements $a, b \in A$ such that $(a, b) \in \operatorname{Com}^{o}\left(f_{\max }\right) \cap E(R),(b, a) \notin$ $\operatorname{Com}^{o}\left(f_{\max }\right) \cap E(R),\left.\phi^{\prime \prime}\right|_{\{a, b\}},\left.\psi^{\prime \prime}\right|_{\{a, b\}}$ are both commutative. If $(a, b)$ is a vertex in $T_{F}^{R}$, then one of $\phi^{\prime}, \psi^{\prime}$ satisfies $\stackrel{a}{\downarrow}$, and therefore it holds for one of $\phi^{\prime \prime}, \psi^{\prime \prime}$, too. If $(a, b)$ is not a vertex in $T_{F}^{R}$, i.e. $\{a, b\} \notin M$, then $\underset{b}{\underset{b}{\downarrow} \phi^{\prime \prime}, \underset{b}{\downarrow} \psi^{\prime \prime}}$, because $\left.F\right|_{\{a, b\}}$ contains only one commutative operation. In both cases the second property of a weak tournament pair on $\operatorname{Com}^{o}\left(f_{\max }\right) \cap E(R)$ is satisfied.

In case that $(a, b),(b, a) \notin \operatorname{Com}^{o}\left(f_{\max }\right) \cap E(R)$, we see that $\{a, b\} \notin$ $\operatorname{Com}\left(f_{\max }\right)$ (the case when $\{a, b\} \in \operatorname{Com}\left(f_{\max }\right),(a, b),(b, a) \notin E(R)$ is impossible due to completeness of $U G(R))$ which means that $\phi_{\{a, b\}}^{\prime \prime}, \psi_{\{a, b\}}^{\prime \prime}$ are projections. Thus, a pair of operations $\phi(x, y)=\phi^{\prime \prime}\left(x, \phi^{\prime \prime}(y, x)\right), \psi(x, y)=$ $\psi^{\prime \prime}\left(\psi^{\prime \prime}(y, x), y\right)$ satisfy all three properties of a weak tournament pair on $\operatorname{Com}^{\circ}\left(f_{\max }\right) \cap E(R)$.

Theorem 9. If $F$ satisfies the necessary local conditions for $R$ and $\overline{\operatorname{Com}\left(f_{\max }\right)} \neq \emptyset$, then $F$ contains an arithmetical operation on $\overline{\operatorname{Com}\left(f_{\max }\right)}$.

Proof. Obviously, for every $B \in \overline{C o m\left(f_{\max }\right)},\left.F\right|_{B}$ cannot contain any commutative binary function. Therefore, every binary function in $\left.F\right|_{B}$ is a projection. 
For $B \in \overline{\operatorname{Com}\left(f_{\max }\right)}$, let $m^{B}$ be an arithmetical function on $B$; existence of this function follows from the necessary local conditions for $R$. Assume now that $\overline{\operatorname{Com}\left(f_{\max }\right)}=\left\{\left\{x_{1}, y_{1}\right\}, \ldots,\left\{x_{s}, y_{s}\right\}\right\}$. We prove by induction that for every $r \leq s, F$ contains a function $m_{r}: A^{3} \rightarrow A$ that is arithmetical on $\left\{\left\{x_{i}, y_{i}\right\} \mid 1 \leq i \leq r\right\}$.

When $r=1, m_{1}(x, y, z)=m^{\left\{x_{1}, y_{1}\right\}}(x, y, z)$ and the statement is obviously true. Suppose it is true for $r \leq k<s$ and that we have the function $m_{k}: A^{3} \rightarrow A$. Let us prove the statement for $r=k+1$. If $m_{k}$ is arithmetical on $\left\{\left\{x_{k+1}, y_{k+1}\right\}\right\}$, then we define $m_{k+1} \triangleq m_{k}$ and the statement is proved. Otherwise, one of the following three statements is true

$$
\begin{aligned}
& \exists x, y \in\left\{x_{k+1}, y_{k+1}\right\}\left[m_{k}(x, x, y) \neq y\right], \\
& \exists x, y \in\left\{x_{k+1}, y_{k+1}\right\}\left[m_{k}(y, x, x) \neq y\right], \\
& \exists x, y \in\left\{x_{k+1}, y_{k+1}\right\}\left[m_{k}(y, x, y) \neq y\right] .
\end{aligned}
$$

Suppose the first case holds (the proof for other cases is analogous), i.e. $\left.m_{k}\right|_{\left\{x_{k+1}, y_{k+1}\right\}}(x, x, y)$ is the $x$-projection. It is easy to see that the function $m_{k+1}(x, y, z)=m_{k}\left(m^{\left\{x_{k+1}, y_{k+1}\right\}}(x, y, z), m^{\left\{x_{k+1}, y_{k+1}\right\}}(x, y, z), m_{k}(x, y, z)\right)$ is arithmetical on $\left\{\left\{x_{i}, y_{i}\right\} \mid 1 \leq i \leq k+1\right\}$.

Induction completed and it is clear that $m_{s}(x, y, z)$ satisfies the condition of theorem.

\section{Proof of Theorem 5}

Suppose we have some instance $(V, C, W)$ of $\operatorname{MinHom}_{R}(\operatorname{Inv}(F))$. Let $\operatorname{Sol}(V, \mathrm{C}, W)$ denote the set of its solutions. By Theorem 8 , there is a pair $\phi, \psi \in F$ which is a weak tournament pair on $\operatorname{Com}^{o}\left(f_{\max }\right) \cap E(R)$.

Until the end of this section, the pair $\phi, \psi$ will be fixed. For any $f, g$ : $V \rightarrow A$, define $\phi(f, g), \psi(f, g): V \rightarrow A$ such that $\phi(f, g)(v)=\phi(f(v), g(v))$, $\psi(f, g)(v)=\psi(f(v), g(v))$. Since any constraint relation of $\mathrm{C}$ is preserved by $\phi, \psi$, we have $\phi(f, g), \psi(f, g) \in \operatorname{Sol}(V, \mathrm{C}, W)$ for $f, g \in \operatorname{Sol}(V, \mathrm{C}, W)$.

Lemma 2. For any measure $M(f)=\sum_{v \in V} \sum_{r \in R} w_{v r} r(f(v))$ we have

$$
M(\phi(f, g))+M(\psi(f, g)) \leq M(f)+M(g)
$$

Proof. Let us prove that for any $a, b \in A$ and any $r \in R: r(a)+r(b) \geq$ $r(\phi(a, b))+r(\psi(a, b))$.

If $(a, b),(b, a) \in \operatorname{Com}\left(f_{\max }\right) \cap E(R)$ or $\{a, b\} \notin \operatorname{Com}\left(f_{\max }\right)$, then $\{\phi(a, b), \psi(a, b)\}=\{a, b\}$, and we have an equality. If $(a, b) \in \operatorname{Com}\left(f_{\max }\right) \cap$ $E(R),(b, a) \notin E(R)$, then $\{\phi(a, b), \psi(a, b)\}=\{a, b\}$ or $\{\phi(a, b), \psi(a, b)\}=$ $\{b\}$. In the first case we have an equality and in the second case we have:

$$
\forall r \in R, r(a)+r(b) \geq 2 r(b) \Leftrightarrow \forall r \in C r(b) \leq r(a) \Leftrightarrow(b, a) \notin E(R)
$$


From this inequality, we conclude that for any measure $M(f)=$ $\sum_{v \in V}^{n} \sum_{r \in R} w_{v r} r(f(v))$ we have $M(f)+M(g) \geq M(\phi(f, g))+M(\psi(f, g))$

Lemma 3. Suppose $A_{v}=\{f(v) \mid f \in \operatorname{Sol}(V, C, W)\}$ and there is $a, b \in A_{v}$ such that $\underset{b}{\downarrow} \psi, \underset{b}{\downarrow} \phi$. Then we have

$$
\min _{f \in \operatorname{Sol}(V, \mathrm{C}, W), f(v)=b} M(f) \leq \min _{f \in \operatorname{Sol}(V, \mathrm{C}, W), f(v)=a} M(f)
$$

Proof. Suppose that $f^{*} \in \operatorname{Sol}(V, \mathrm{C}, W), f^{*}(v)=a$ and $f^{* *} \in$ $\operatorname{Sol}(V, \mathrm{C}, W), f^{* *}(v)=b$ such that

$$
M\left(f^{*}\right)=\min _{f \in \operatorname{Sol}(V, \mathrm{C}, W), f(v)=a} M(f), M\left(f^{* *}\right)=\min _{f \in \operatorname{Sol}(V, \mathrm{C}, W), f(v)=b} M(f)
$$

Since $\phi\left(f^{*}, f^{* *}\right)(v)=b, \psi\left(f^{*}, f^{* *}\right)(v)=b$, then by Lemma 2

$$
2 M\left(f^{* *}\right) \leq M\left(\phi\left(f^{*}, f^{* *}\right)\right)+M\left(\psi\left(f^{*}, f^{* *}\right)\right) \leq M\left(f^{*}\right)+M\left(f^{* *}\right)
$$

Now

are ready to describe polynomial-time algorithm for $\operatorname{MinHom}(\operatorname{Inv}(F))$, which conceptually follows the proof of Theorem 8.3 in [3]. First we compute sets $A_{v}=\{f(v) \mid f \in \operatorname{Sol}(V, \mathrm{C}, W)\}$ for every $v \in V$. This could be done polynomialtime, because, by result of [2], $C S P(\operatorname{Inv}(F))$ is polynomial-time solvable by 3-consistency algorithm: adding a constraint $(v,\{a\})$ to $\mathrm{C}$ we can find whether $a \in A_{v}$ or not. After this operation, we iteratively delete all elements $a$ from every $A_{v}$ such that there is $b \in A_{v}: \underset{b}{\downarrow} \psi \underset{b}{\downarrow} \phi$. This operation will not increase a minimum of optimized functional due to Lemma 3. Afterwards, we update every constraint pair $\left(\left(v_{1}, \ldots, v_{m}\right), \rho\right) \in \mathrm{C}$ by defining $\rho=\rho \cap A_{v_{1}} \times \ldots \times A_{v_{m}}$.

Consider a collection of sets $\mathrm{A}=\left\{A_{v} \mid v \in V\right\}$. Obviously, $|\mathrm{A}|<2^{|A|}$. For a pair $\left(\left(v_{1}, \ldots, v_{m}\right), \rho\right) \in \mathrm{C}$, a predicate $\rho$ can be considered as multi-sorted predicate over $\mathrm{A}$ with signature $\left(A_{v_{1}}, \ldots, A_{v_{m}}\right)$. Moreover, for any $p \in F$ this multi-sorted predicate preserves a polymorphism $\left\{p^{B} \mid B \in \mathrm{A}\right\}$ where $p^{B}=\left.p\right|_{B}$.

By

Theorem 9, $F$ contains an operation $m$ which is arithmetical on $\overline{\operatorname{Com}\left(f_{\max }\right)}$. Consider multi-sorted polymorphisms $\left\{\phi^{B} \mid B \in \mathrm{A}\right\},\left\{\psi^{B} \mid B \in \mathrm{A}\right\},\left\{m^{B} \mid B \in \mathrm{A}\right\}$. It is easy to see that this polymorphisms satisfy conditions of Theorem 7 and the set of multi-sorted predicates $\{\rho \mid(s, \rho) \in \mathrm{C}\}$ is tractable. Therefore, we can polynomially solve our problem.

\section{Directions for further work}

As it was said in the introduction, $\mathrm{MinHom}_{R}$ fits the framework of Valued CSP $(V C S P)$ [3]. The following subproblems of VCSP generalize MinHom and are not investigated yet. 
1. What happens with $\mathrm{MinHom}_{R}$ when $U G(R)$ is not complete. We believe that Theorem 5 is true for this case too.

2. Suppose we have a finite valued constraint language $\Gamma$, i.e. a set of valued predicates over some finite domain set. If $\Gamma$ contains all unary valued predicates, we call $\operatorname{VCSP}(\Gamma)$ a conservative $\operatorname{VCSP}$. This name is motivated by the fact that in this case the multimorphisms (which is a generalization of polymorphisms for valued constraint languages [3]) of $\Gamma$ must consist of conservative functions. Since there is a well-known dichotomy for conservative CSPs [2], we suspect that there is a dichotomy for conservative VCSPs.

\section{References}

1. Bodnarcuk, V.G., Kalužnin, L.A., Kotov, N.N., Romov, B.A.: Galois theory for Post algebras. Kibernetika 3 1-10, 5 1-9 (in Russian)

2. Bulatov, A.A.: Tractable conservative Constraint Satisfaction Problems. 18th Annual IEEE Symposium on Logic in Computer Science (2003) 321-330

3. Cohen, D., Cooper, M., Jeavons, P.: Generalising submodularity and horn clauses: Tractable optimization problems defined by tournament pair multimorphisms. Theor. Comput. Sci. 401 (2008) 36-51

4. Daniels, H., Velikova, M.: Derivation of monotone decision models from nonmonotone data. Tilburg University, Center Internal Report 30 (2003)

5. Feder, T., Vardi, M. Y.: The computational structure of monotone monadic SNP and constraint satisfaction: A study through datalog and group theory. SIAM Journal on Computing 28 (1999) 57-104

6. Geiger D.: Closed Systems of Functions and Predicates. Pacific Journal of Mathematics 27 (1968) 95-100

7. Gupta, A., Hell, P., Karimi, M., Rafiey, A.: Minimum cost homomorphisms to reflexive digraphs. LATIN (2008)

8. Gutin, G., Hell, P., Rafiey, A., Yeo, A.: A dichotomy for minimum cost graph homomorphisms. European Journal of Combinatorics 29 (2008) 900-911

9. Gutin, G., Hell, P., Rafiey, A., Yeo, A.: Minimum cost and list homomorphisms to semicomplete digraphs. Discrete Appl. Math. 154 (2006) 890-897

10. Gutin, G., Rafiey, A., Yeo, A., Tso, M.: Level of repair analysis and minimum cost homomorphisms of graphs. Discrete Applied Mathematics 154 (2006) 881-889

11. Gutin, G., Rafiey, A., Yeo, A.: Minimum Cost Homomorphism Dichotomy for Oriented Cycles. Proceedings of AAIM'08. LNCS 5034 (2008) 224-234

12. Jeavons P.: On the Algebraic Structure of Combinatorial Problems. Theoretical Computer Science 200 (1998) 185-204

13. Jonnson P.: Boolean constraint satisfaction: complexity results for optimization problems with arbitrary weights. Theoretical Computer Science 244, 1-2, pp. 189$203(2000)$

14. Jonsson, P., Kuivinen, F., Nordh, G.: Max Ones generalised to larger domains. SIAM Journal on Computing 38 (2008) 329-365

15. Jonsson, P., Nordh, G.: Generalised integer programming based on logically defined relations. Mathematical Foundations of Computer Science 2006. LNCS 4162 (2006) 549-560

16. Jonsson, P., Nordh, G., Thapper, J.: The maximum solution problem on graphs. Mathematical Foundations of Computer Science 2007. LNCS 4708 (2007) 228-239 
17. Marchenkov, S.S.: Closed classes of boolean functions. Nauka, Fizmatlit, Moscow (2000) (in Russian)

18. Post, E.: The two-valued iterative systems of mathematical logic. Annals of Mathematical Studies 5 (1941)

19. Rafiey, A., Hell, P.: Duality for Min-Max Orderings and Dichotomy for Min Cost Homomorphisms, http://arxiv.org/abs/0907.3016v1

20. Schaefer, T.J.: The complexity of satisfiability problems. 10th ACM Symposium on Theory of Computing (1978) 216-226

21. Takhanov, R.: A dichotomy theorem for the general minimum cost homomorphism problem. STACS (2010) (to appear) 